Lee, S.L. (2012). The effects of gesture-based learning with virtual reality on counting ability for children with cerebral palsy. Journal of Educational Technology Development and Exchange, 5(1), 109-118.

\title{
The Effects of Gesture-based Learning with Virtual Reality on Counting Ability for Children with Cerebral Palsy
}

\author{
Shwu-Ling Lee \\ Taipei University of Education
}

\begin{abstract}
This study investigated the effects of gesture-based learning with virtual reality (GBLVR) on the counting ability of three children with Cerebral Palsy (CP). Those elected were the children who had difficulty with counting skills in the classroom. By attending GBLVR math courses, children with CP learned counting numbers from 1 to 10 intuitively by moving their body and interacting with the design made for them. The single-subject experimental design within the multiple probe design across subjects was used to evaluate the effects. Virtual Reality Gesture-based Learning was the independent variable; the score from the Counting Ability was the dependent variable. The results of this study show an instant effect, a maintenance effect, and a generalization effect of the score of Counting Ability were found.
\end{abstract}

Keywords: children with Cerebral Palsy (CP), gesture-based learning with virtual reality (GBLVR), counting ability

\section{Introduction}

Children with Cerebral Palsy (CP) may have different sorts of disability problems. It is possible that their physical mobility is a problem, but their vision may be developing as well. This physical disability causes to reduce the tactile information received. They have tactile information, but they cannot reach beyond a very small area and they cannot react in the same way as able-bodied children. The degree of interaction between touch and vision from children with $\mathrm{CP}$ is limited, due to the fact that their comprehension is built from vision only. Therefore, the intervention in this study was to provide them with the means to integrate sensory information and find an appropriate feedback mechanism. The aim here was to find whether they can then deal with number concepts.

Children with CP tend to have the following symptoms. First, the spastic symptom makes them rigid in movement and they have the inability to relax muscles. The extent of the disability varies. Some muscles become very stiff and weak, especially when under pressure (Stopford, 1988). They even fail to touch the keyboard and control the mouse. Second, the athetoid symptom shows frequent involuntary movements, which mask and interfere with the normal movements of the body. It also causes writhing movement of the limbs, face, and tongue, and also dribbling, grimacing, and slurred speeches. Damage to the basal ganglia of the brain appears to be the 
cause of this condition. People with athetoid tend to make unwanted movements and have some loss of control of their posture (Kirk \& Gallagher, 1979). Their fluctuated unstable muscle tone, uncoordinated movements, poor eye-hand coordination, and over twisted action will often cause error while pressing buttons or number keys. Third, the ataxia symptom show difficulties in hand and eye coordination control causing poor body balance and unsteady gait. Children with ataxic frequently have irregular and shaky hand movements (Stopford, 1988). This shivering causes poor fine motor skills, causing trouble in typing. Due to the reasons above, computer assistive technology is very helpful in learning acquisition for students with physical disabilities. Liu (2001) confirmed the effects of assistive technology for students with CP. It can solve learning problems and increase the effectiveness of learning. However, the computer interface for students with $\mathrm{CP}$ is still a great challenge if applicable hardware and software are only developed for these special populations. Due to the limited market of individuals with special needs, willingness of manufacturers to improve the design of assistive technology is very low (King, 1999).

When children with $\mathrm{CP}$ were provided with the appropriate means of feedback, they did learn to integrate their action with vision in order to promote their counting ability. Gesture-based Learning with Virtual Reality (GBLVR) can give users a more immersive and natural feeling in the manipulation and interaction with virtual objects. The possibility to use GBLVR to open up access is based on an application of theory of perceptual development.

One method of intervention currently receiving widespread attention is the use of Gesture-based Learning with Virtual Reality systems for rehabilitation purposes. These systems demand focus and attention, can motivate the user to move, and provide the user with a sense of achievement, even if they cannot perform that task in the 'real world.' Reid (2002) presents the results of a clinical trial of a virtual reality play-based intervention. The results of three single case studies are presented. The gesture-based learning with virtual reality intervention used the Mandala Gesture Xtreme technology. It was applied to three school-aged children with cerebral palsy. A pre-test/post-test design was used. Group scores on the Canadian Occupational Performance Measure (COPM) indicated clinically significant changes in selfefficacy for all children. Two of the participants demonstrated the greatest changes in both perceived performance abilities and task specific domain areas with satisfactory scores. Qualitative comments from the participants revealed a high degree of motivation, interest, pleasure, and opportunity for engagement in play; activities that were not previously engaged in. Overall, this pilot study suggests the viability of a virtual reality play-based intervention as part of the rehabilitation process for children with cerebral palsy. Several studies suggest that VR game-based technology can be used to improve motor skill rehabilitation of functional deficits including hand function (Broeren, et al., 2002; Holden, Todorov, Callaban, \& Bizzi, 1999; Merians, et al., 2002; Reid, 2002a Wann \& Turnbull, 1993) and walking (Baram \& Miller, 2006; Fulk, 2005; Fung, Malouin et al 2004; Fung, Richards et al 2006). However, clinic and home-based systems need to be affordable and easy to deploy and maintain, while still providing the interactional fidelity required to produce the meaningful motor rehabilitation activity needed to foster transfer to the real world.

In fact, several studies suggest that GBLVR could provide a controlled and convenient environment where various abilities could then be accessed and be of access. Some studies proposed evidences that 
investigated the effects of gesture-based in a virtual environment on way finding (Hirose, et al., 1994; Inman, et al., 1997; Lue \& Lee, 2008; Nemire \& Crane, 1995; Muscott \& Gifford, 1994; Reid, 2002a, b; Rose, Attree, \& Johnson, 1996; Stephenson, 1994; Wilson, et al., 1996).

Liu (2010) created two physical computer version interactive games - "Little Chef" and "Recycle" - for autistic children. By playing games, autistic children intuitively move their whole body and learn cognitive courses in these games. After the experiment of behavioural observations and evaluations, the results show that autistic children can develop their behavioural management and increase their cognitive knowledge at the same time in these GBLVR games.

There were several more studies (Hung, 2008; Lee, \& Huang, 2007; Lin, 2008; McComas, MacKay \& Pivik, 2002) that showed through GBLVR, the participants revealed significant changes in self-efficacy. However, these studies did not examine the effects on cognitive skills of children with CP. There is a need for further examination on the effects of cognitive development (e.g., Counting skills) for children with CP. Testing counting skills in GBLVR learning environment is often preferred over a traditional classroom; this trend is becoming increasingly popular because GBLVR environment represent a controlled and convenient environment where various abilities, including those children with $\mathrm{CP}$, can access and be assessed. The aim of the study is to investigate the effects of GBLVR on the counting ability of three children with Cerebral Palsy.

The following are research questions used in this study to investigate this area:
(1) How is the instant effect of GBLVR on the counting ability for three children with CP?

(2) How is the maintenance effect of GBLVR on the counting ability for three children with CP?

\section{Method}

\subsection{Participants and Setting}

Three children with cerebral palsy participated in the study. Participant A was a 5-year-old boy. Participant B was a 4.5 -yearold boy. Participant $\mathrm{C}$ was a 5-year-old girl. The participants displayed difficulty on counting abilities and gross motor skills. All sessions were conducted in a classroom at a pre-school.

\subsection{Materials}

The materials used in this study included a notebook with a built in camera, GBLVR software for counting teaching sessions, a video camera, and a list of degree of prompts used by the researcher to respond to participants' prompts during the gesture-based play. Each GBLVR had three parts which include Teaching, Practice, and Testing.

\section{Part I: Teaching}

Saying numbers in sequence: In order to count from 1 to 5 in sequence, the ball changes color when touched and the number is printed on the yellow banner below. In addition, the number of the ball is called out in voice. After counting 1-5, the numbers change to 6-10. When one has finished counting all 10 numbers, the screen changes back to 1-5. All children can play the game rapidly. 


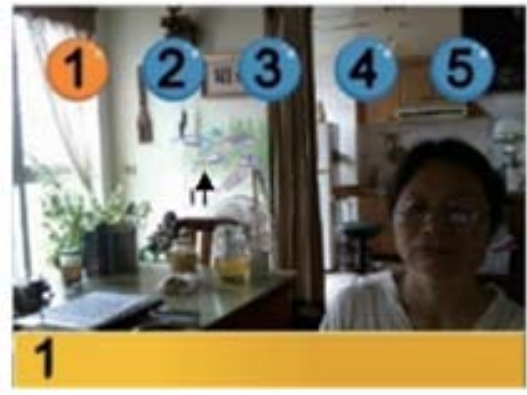

\section{Number from left to right}

Rational counting: This is an extension of point counting. Students learn to apply the cardinality rule: The last number said after counting informs students how many there are in a set. When the child points at an object (e.g., a cow), it changes to a ball with a number and pronounces 'there are 10 cows.' The children can play through it rapidly.

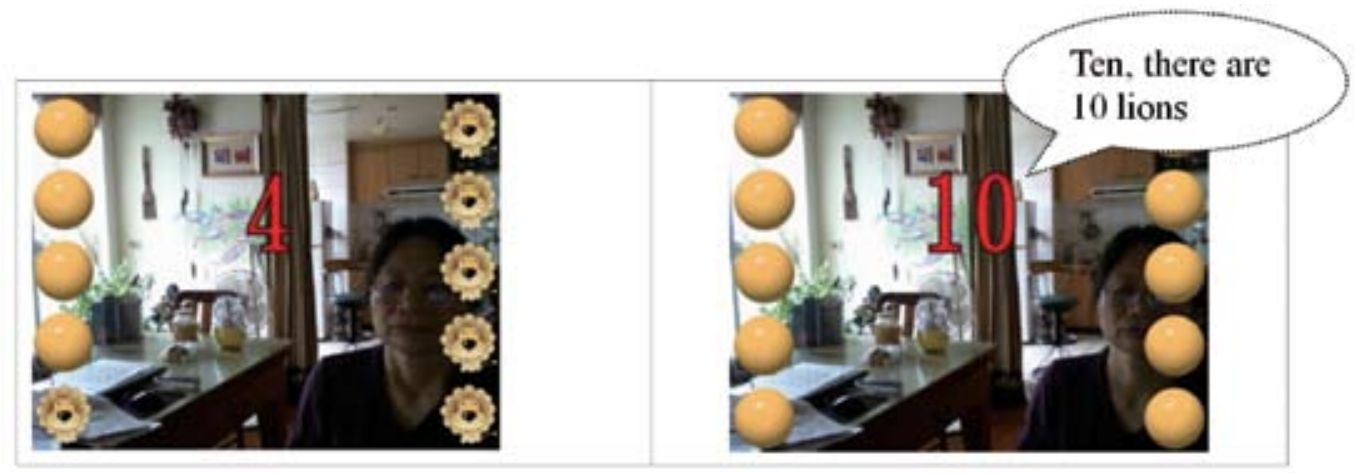

\section{Part II: Practice}

Saying numbers in sequence: Numbers display on the screen in random, and after the child counts $1-5$, the screen will change to $6-10$. Children can play this game again and again.
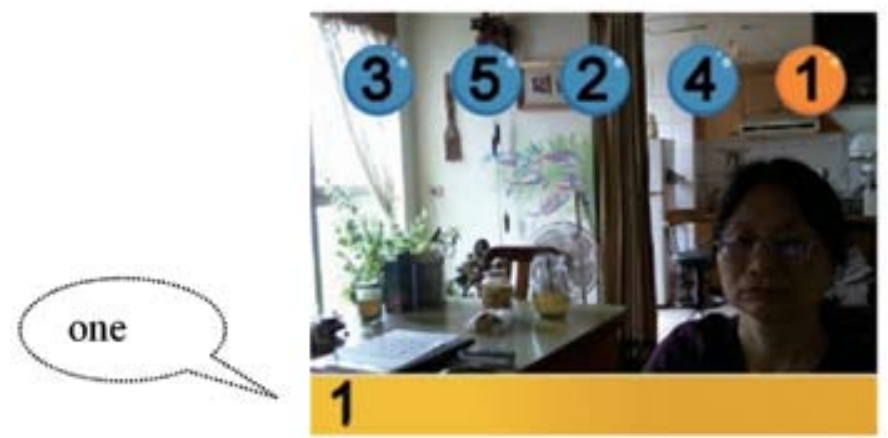

Number from left to right 
Rational counting: When different animals are displayed in different situations, it will ask the child "how many animals are there?" When the child chooses the number, the screen will provide a feedback such as "Correct" or "There are 3 hens, please try again."

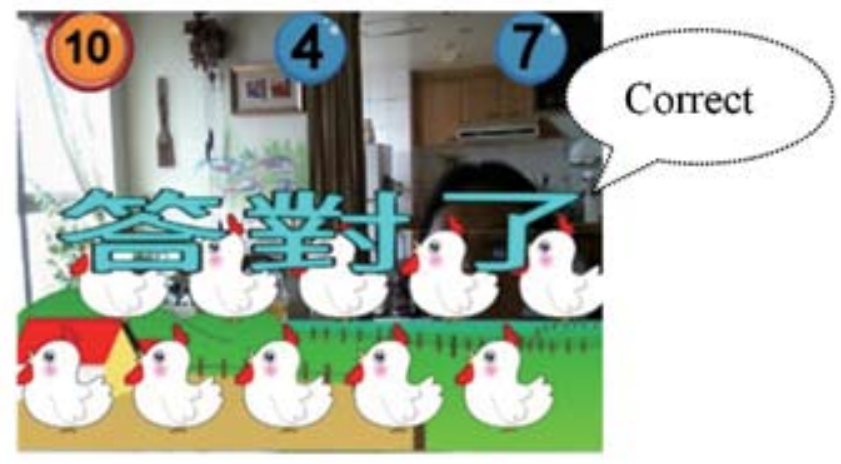

Part III testing: There are 10 questions (number 1-10 will display on the screen in random). If correct, each question gets a 10 point score on the screen and gives a feedback such as "Correct." If wrong, it will only get a feedback (i.e., "Wrong" with no answer to the question). After 10 questions, the screen will display the total score.

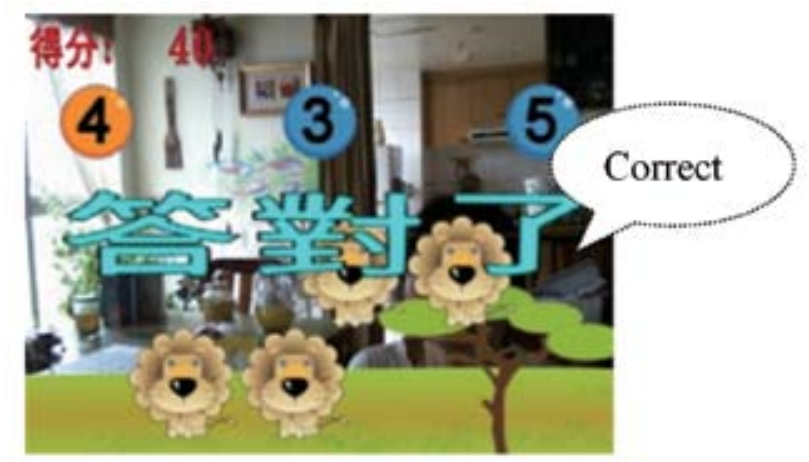

\subsection{Data Collection and Analysis}

During all assessment and treatment sessions, videos were recorded. Two trained observers watched the videos and recorded the score of counting ability on the screen after testing (Part III).

Inter-observer agreement was calculated for all trials (for all participants) using the total count-per-trial method after the testing. The researchers divided the number of trials in which an agreement was scored (i.e., the two observers recorded the score of counting ability) by the total number of trials (agreements plus disagreements), and this ratio was converted to a percentage. Across all participants, inter-observer agreement averaged 100\%. Data obtained from the blind coding process were analyzed using standard 
visual inspection procedures (Barlow, Nock, \& Hersen, 2009).

\subsection{Procedure}

Experimental design. An ABA design was used to evaluate to what extent counting ability could be acquired through Virtual Reality Gesture-based Learning. Data sets were arranged in a three-tier non-concurrent multiple baseline design across three participants.

Pre-experiment training. Prior to the study, participants were trained on the GBLVR by teachers. The training included one or both of the following: verbal instructions and/or modeling on GBLVR.

Baseline (A). During baseline, participants had access to GBLVR from prior training. The data collector recorded the score of the participant to engage the "Part III Test" in GBLVR exercise using his / her part of the body. During the GBLVR, no "Part I Teaching" and "Part II Practice" of GBLVR were provided.

Treatment probes (B). During the treatment phase, conditions were identical to baseline except that the participant played GBLVR from Part I Teaching to Part II Practice, and then the researcher recorded the score after doing the Part III Test.

Maintenance probes (M). A maintenance probe was conducted for each participant after the performance criterion (i.e., $90 \%$ or more correct) had been met for three consecutive GBLVR sessions. Maintenance probes were conducted identically to the baseline. The researcher recorded the score after doing the Part III Test.

Generalization probes (G). The generalization probe was conducted with each participant during baseline and maintenance conditions. During probe sessions with real situation, the data collector asked the child to count the real objects provided.

\section{Results and Discussion}

\subsection{Results}

Results of the GBLVR for participants are presented in Figure 1. Baseline scores of responding to counting ability were low, with a mean of 2.5 for Participant 1 , a mean of 0 for Participant 2, and a mean of 2 for Participant 3. When the GBLVR sessions were implemented, responding to the counting skill for three participants was high; however, a slight rising trend can be detected across the condition. Responding to the counting skill during this GBLVR treatment increased from baseline to a mean of 81.43 for Participant 1 , a mean of 80 for Participant 2, and a mean of 78.5 for Participant 3. Thus, a marked rising trend across the GBLVR treatment has occurred.

At the maintenance phase, each participant sustained high levels of counting item completion after the GBLVR intervention was withdrawn. The mean score of test items completed correctly was still 100 , even after the withdrawal of treatment for Participant 1, Participant 2, and Participant 3. Children with CP did maintain their performance at all the levels. At the generalization phase, each participant similar to the conditions during baseline and maintenance was conducted. But, during the generalization phase it is experimented with real situations. The results were that all children could count the real objects provided with no error (score was 100).

The researchers saw little improvement in counting ability during baseline phase; while there was a large increase in counting scores during the intervention phase. There was a significant progress during the intervention 
period in children's counting ability, but there was no significant change during the baseline phase. This is because the intervention phase (GBLVR) had more effect on self-learning practice (baseline) due to its intervention (GBLVR) being more effective than the self-practice (baseline). When training was terminated, three participants' scores remained high during maintenance probes and generalization probes. In other words, children had already learned counting ability during the intervention (GBLVR).

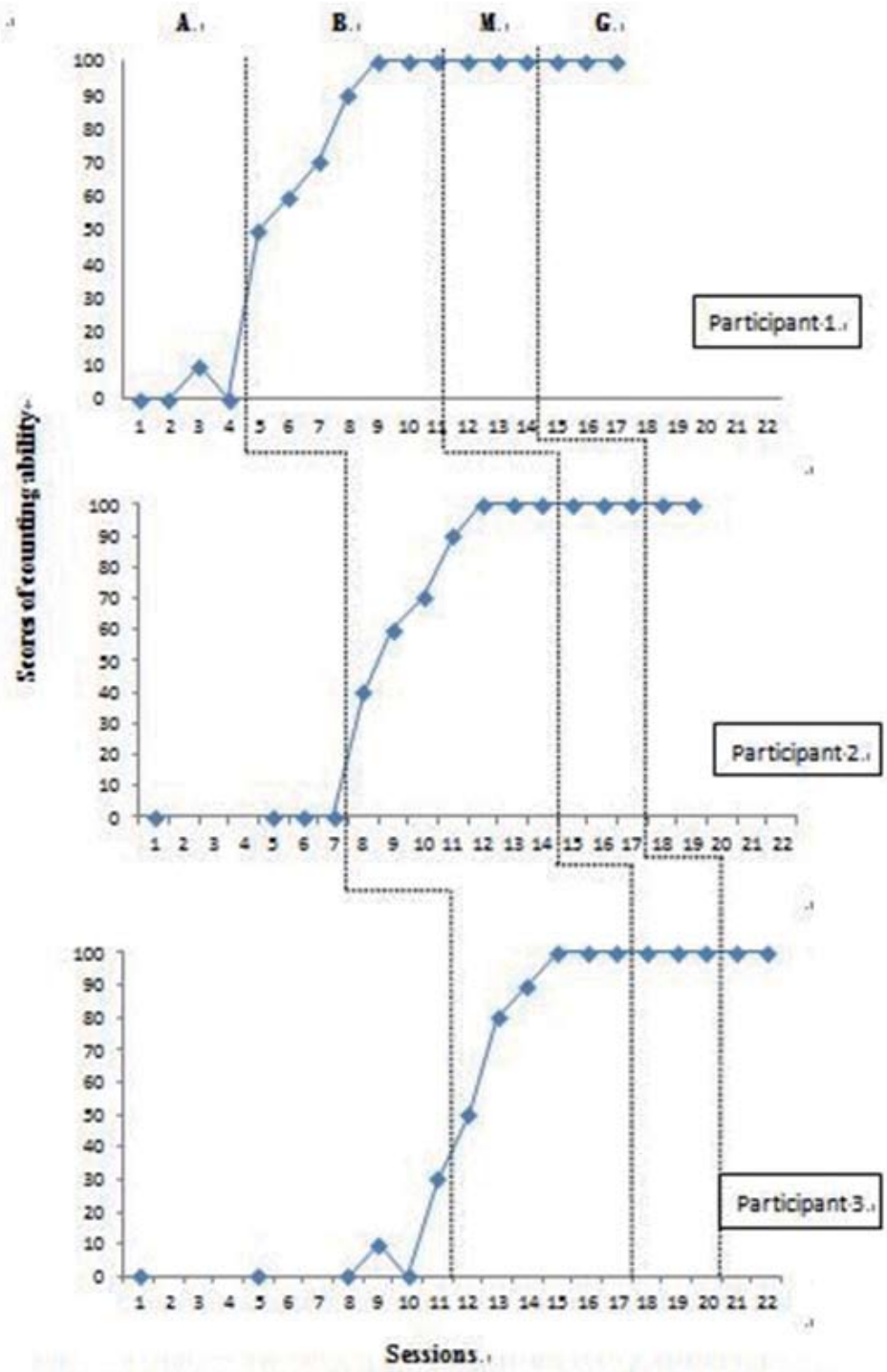

Figure 1 - The effects of -GBLVR on counting ability for 3 participants. 


\subsection{Discussion}

This study demonstrates the effectiveness of intervention. Children were able to produce more interest in counting using GBLVR. The study indicates that counting (1-10) targets are attainable if the right circumstances and tools are supplied. At the same time, the study supports the view that integration of visual and tactile components are critical components.

Lewis (1987) claimed that cognitive development cannot occur in the absence of action and a variety of different types of actions are necessary. Doing without, the child may develop a different perception that can be less effective. It can be argued that because children with $\mathrm{CP}$ have difficulty with their mobility, they may develop an altered perception. This may be the explanation of the findings of research such as Wann (1991). In any case, it is of interest to discover how such children deal with math instruction and how they express themselves.

The issue we deal with in this psychological view is what is going on in children's mind and how they express their perception. The prediction is that children have reduced counting analysis and have problems with counting. This will be a major problem if they are taught using traditional tools. Their limited motor control only adds to the problem. The implications are that feedback from experience does not work effectively. These pupils build up a limited perception, which then, in turn, can alter later understanding. We have to understand this difference in perception if we are to develop an appropriate teaching approach.

Participants were still limited by their motor control problems in operating simple objects and expressing counting ability. Thus, it has been shown that there is ineffective learning in ordinary math teaching. These results are like Cruckshank et al's (1969) study that also showed that inappropriate tools could not support the reality perception of the children. However, in the intervention period, children with $\mathrm{CP}$ could acquire the proprioception information to be able to demonstrate improvement in performance. By manipulating images during GBLVR, they could learn counting skills. By controlling directly physical interaction with images on the screen, they could improve their counting ability. Same was true in the study of Van der Weel, Van der Meer, and Lee (1996) where concrete tasks produced better performances; learners have greater information support from the environment.

This meant that children with CP lacked these two factors. They not only have limits of muscular activity, but also lack the ability to express their perception. In the study, firstly, GBLVR was provided to overcome the limitation of muscular activity. Secondly, they were instructed in math teaching on how to perform the act. The outcome was that integration occurred and progress in counting was recorded. The children could now be taught.

\section{Conclusion}

The study created an environment where pupils with $\mathrm{CP}$ could improve their perception and express their idea through GBLVR. In math education, GBLVR could be of great value to children with $C P$ in enhancing their development of counting perception. This is consistent with Lewis (1987) who indicated that children with severe physical disabilities can develop cognitively and can have an understanding of objects, provided that they have some means of acting on the environment. These children may not be able to interact with objects in a conventional way, but they may still reach a level of understanding similar to able-bodied children, although by an alternative route. 
When children with CP were provided with the appropriate means of feedback, they did learn to integrate their action with vision in order to promote their counting ability. Although this research involved only a small group of children $(n=3)$ who had limited experience and access to math in pre-school, the results imply that there is much to be gained from GBLVR instructing them in counting.

\section{Reference}

Baram, Y. \& Miller, A. (2006). Virtual reality cues for improvement of gait in patients with multiple Sclerosi., Neurology, 66(2), 178-81.

Barlow, D. H., Nock, M. K., \& Hersen, M. (2009). Single case experimental designs: Strategies for studying behavior change (3rd ed.). Boston: Pearson Education.

Fulk, G. D. (2005). Locomotor training and virtual reality-based balance training for an individual with multiple sclerosis: a case report. J Neurol Phys Ther, 29(1), 34-42.

Fung, J., Malouin, F. et al (2004). Locomotor rehabilitation in a complex virtual environment. Conf ProcIEEE Eng Med Biol Soc, 7, 4859-61.

Fung, J., Richards, C. L. et al (2006). A treadmill and motion coupled virtual reality system for gait training post-stroke. Cyberpsychol Behav, 9(2), 157-62.

Hirose, M., Taniguchi, M., Nakagaki, Y., \& Nihei, K. (1994). Virtual playground and communication environments for children. IEICE Transactions on Information and Systems, E77D, 12, 1330-4.

Hung, W. S. (2008). The effective research of virtual reality in travel skillsTaking vocational school students with intellectual disabilities as an example. (Unpublished master's thesis). National Taipei University of Education.

Inman, D. P., Loge, K., \&Leavens, J. (1997).
Virtual reality education and rehabilitation. Communications of the ACM 40, 53-58.

King, T. M. (1999). Assistive technology: Essential human factors. Boston,MA: Allyn and Bacon.

Kirk, S. A., Gallagher, J. J., \& Anastasiou, N. J. (1993). Educating Exceptional Children. (7th ed.). Boston, MA: Houghton Mifflin.

Lee, S. L. \& Huang, C. Y., $(2007,11)$. The Effects of 3-D Graphic-Based Virtual Reality on the Pedestrainized Skills for Elementary Students with Intellectual, Paper presented at The 18th Asion Conference on Mental Retardation, Taipei.

Lee, S. L. (2009). The Influence of Virtual Reality Play in Multisensory Environment on Motivation of Students with Intellectual Disabilities. Oral presented at the 19th Asian Federation on Intellectual Disabilities Conference, Singapore, 22-26 November 2009.

Lin, C. K. (2008). The effective research of virtual reality in elementary intellectual disabilities training of campus wayfinding (Unpublished master's thesis). National Taipei University of Education.

Liu, H. C. (2001). Computer Access Technology for Students with Cerebral Palsy (Unpublished master's thesis). National Kaohsiung Normal University.

Liu, M. N. (2008). A Research of Interactive Physical Game for Autism Children Learning. (Unpublished master's thesis). National Taipei University of Technology.

Liu, S. W. \& Lee, S. L. (2008). The Comparative Study of Traditional Computer Games and Virtual Reality Games on Game-playing Motivation of Children with Cancer in Homebound Education. Journal of Special Education, 28, 97-122.

McComas, J., MacKay, M., \& Pivik, J. (2002). Effectiveness of virtual reality for teaching pedestrian safety. Cyberpsychology and Behavior, 5(3), 185-190. 
Muscott, H. S. \& Gifford, T. (1994). Virtual reality and social skills training for students with behavioral disorders: Applications, challenges and promising practices. Education and Treatment of Children, 17, 417-34.

Nemire, K. \& Crane, R.(1995). Designing a virtual science laboratory to accommodate needs of students with cerebral palsy. In Proceedings of the 1995 CSUN Virtual Reality Conference. Northridge: California State University.

Reid, D. (2002a). The use of virtual reality to improve upper-extremity efficiency skills in children with cerebral palsy: a pilot study. Tech Disability, 14, 53-61.

Reid, D. (2002b). Benefits of virtual play rehabilitation environment for children with cerebral palsy on perceptions of self- efficacy: a pilot study. Pediatric Rehabilitation, 5(3) 141-8.

Rose, F. D., Attree, EA, \& Johnson, D. A. (1996). Virtual reality: An assistive technology in neurological rehabilitation. Current Opinion in Neurology, 9, 461-7.

Stephenson, J. (1994). Sick kids find help in a cyberspace world. Journal of the American Medical Association, 274,
1899-901.

Stopford, V. (1988). Understanding Disability, London: Edward Arnold.

Wilson, P. N., Foreman, N., \& Tlauka, M. (1996). Transfer of special information form a virtual to a real environment in physically disabled children. Disability and Rehabilitation, 18, 633-7.

$\mathrm{Wu}, \mathrm{T}$. F. (2001). Assessments and interventions of computer access for persons with physical disabilities (Unpublished doctoral dissertation). National Taiwan Normal University

\section{Contact the Author}

\section{Shwu-Ling Lee}

Taipei University of Education

Email: bristo10420@gmail.com 